\title{
A CASE OF TWO COMMON CULPRITS CAUSING PULMONARY EMBOLISM
}

\author{
Timothy CTJ', Mansor A $^{2}$, Wengvei CTK ${ }^{3}$ \\ 1 Hospital Lahad Datu, Sabah \\ 2 Faculty of Medicine, University of Malaya \\ 3 Hospital Queen Elizabeth, Sabah \\ Correspondence: \\ Timothy Cheng Tsin Jien \\ Department of Orthopaedics \\ Hospital Lahad Datu \\ 91100 Lahad Datu \\ Sabah \\ Malaysia \\ mail.timc@gmail.com
}

\begin{abstract}
Pulmonary embolism is the obstruction of the pulmonary artery or its branches, commonly by thrombus or fat. We report an unusual case of double pathology - both pulmonary thromboembolism and fat embolism syndrome in a patient with bilateral femur and bilateral tibia fractures. This highlights the importance of a high index of suspicion of these conditions while managing patients with multiple long bone fractures. Morbidity and mortality can be significantly reduced with prompt and appropriate prevention strategies.
\end{abstract}

Keywords: Pulmonary embolism, fat embolism, thromboembolism

\section{Introduction}

Pulmonary embolism is the obstruction of the pulmonary artery or one of its branches by foreign material, namely thrombus, fat, air or tumour. Fat embolism syndrome refers to the presence of fat globules in the lung parenchyma or microcirculation. It is most commonly associated with pelvic and long bone fractures. The risk of developing fat embolism syndrome increases with the number of long bone fractures, which is up to $33 \%$ in patients with bilateral femur fractures (1). Pulmonary thromboembolism denotes the presence of a thrombus within the pulmonary arteries, and its risk factors are based on Virchow's triad, such as prolonged immobilisation, recent surgery, or other causes of hypercoagulability such as malignancy and inherited thrombophilias. We report an unusual case of double pathology, where both pulmonary thromboembolism and fat embolism syndrome were demonstrated in a patient presented with fractures of bilateral femur and tibia.

\section{Case report}

A 21 year-old-man with no significant past medical history was admitted to our hospital 4 hours after a motorcycle accident. He sustained an open fracture (Gustilo Grade II) of the left tibia, open fractures (Gustilo Grade I) of the right femur and tibia, closed fracture of the left femur and closed fractures of the distal ends of both radii. There were no head, neck, chest or abdominal injuries and he was hemodynamically stable upon admission. He was resuscitated with 3 litres of normal saline and 2 pints of whole blood. Wound debridement and external fixation of both tibias were performed 9 hours after the accident, which was delayed due to prolonged resuscitation. He developed hypotension intraoperatively and became unstable; thus, both femurs were not fixed but put on skeletal traction in the ward.

Approximately 42 hours after admission, the patient developed dyspnea with an oxygen saturation of $89 \%$ and $\mathrm{P}_{\mathrm{a}} \mathrm{O}_{2}$ of $56 \mathrm{mmHg}$ on $15 \mathrm{~L}$ of oxygen per minute via high flow mask. He became drowsy and confused, tachycardic and was febrile with a temperature of 38 degree Celsius. His platelet levels were $92 \times 10^{-9} / \mathrm{L}$. A diagnosis of fat embolism was made, and he was electively intubated, ventilated and transferred to the intensive care unit for further management. Axillary, truncal and conjunctival petechiae developed 72 hours after admission (Figure 1). His chest radiograph was clear, and he had no electrocardiogram abnormalities besides sinus tachycardia. 

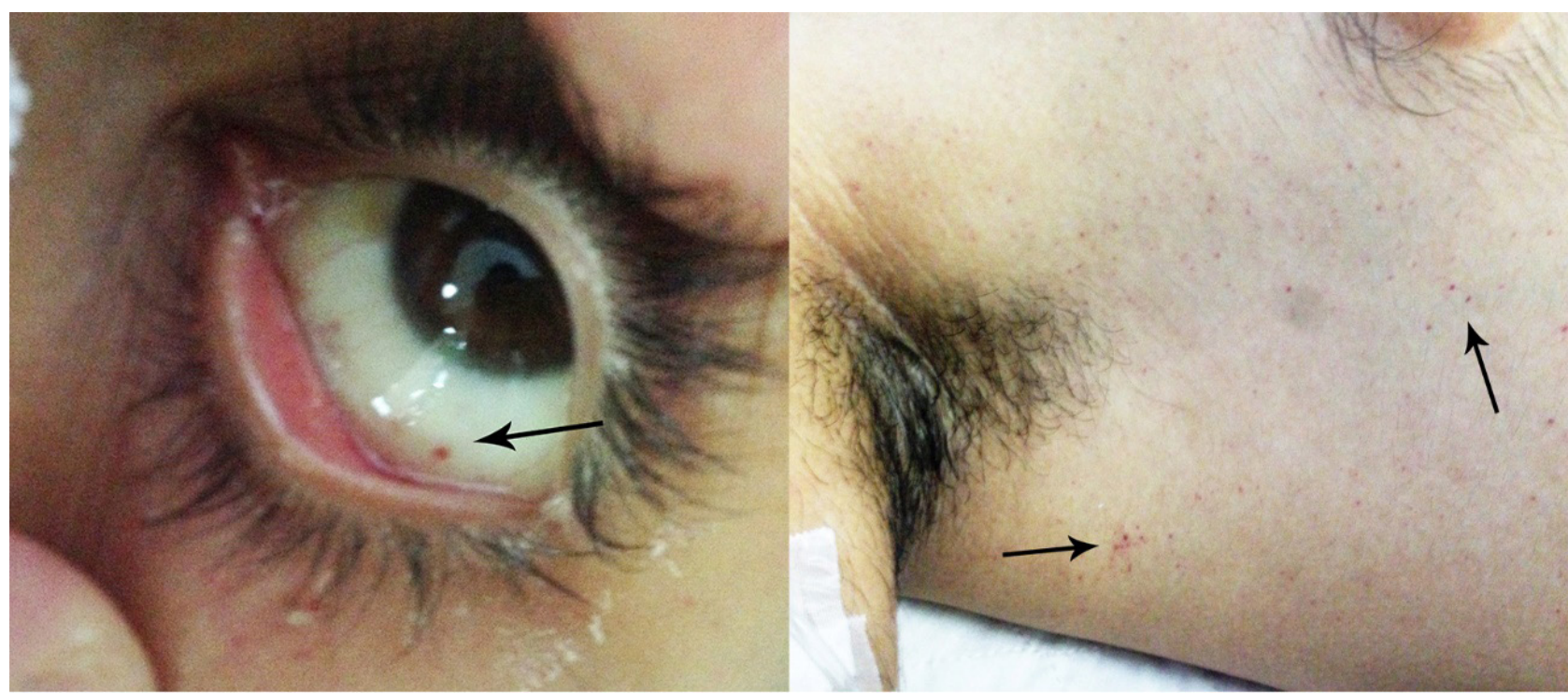

Figure 1: Images showing axillary, truncal and subconjunctival petechiae

A computed tomography pulmonary angiogram was performed to rule out pulmonary thromboembolism. The results revealed a filling defect within the secondary branches of the left descending, right upper lobe pulmonary artery and tertiary branches of descending right pulmonary artery, which indicated the presence of a thrombus (Figure 2). The patient was started on anticoagulation therapy, comprising of subcutaneous enoxaparin $60 \mathrm{mg}$ twice daily.
Subsequently, his femur fractures were plated, and he was extubated after 8 days in ICU and transferred back to the general ward. Both his tibial fractures were fixed with intramedullary nailing two weeks later. Six months post-trauma, he had no neurological sequelae and he is currently ambulating well with crutches while undergoing rehabilitation.

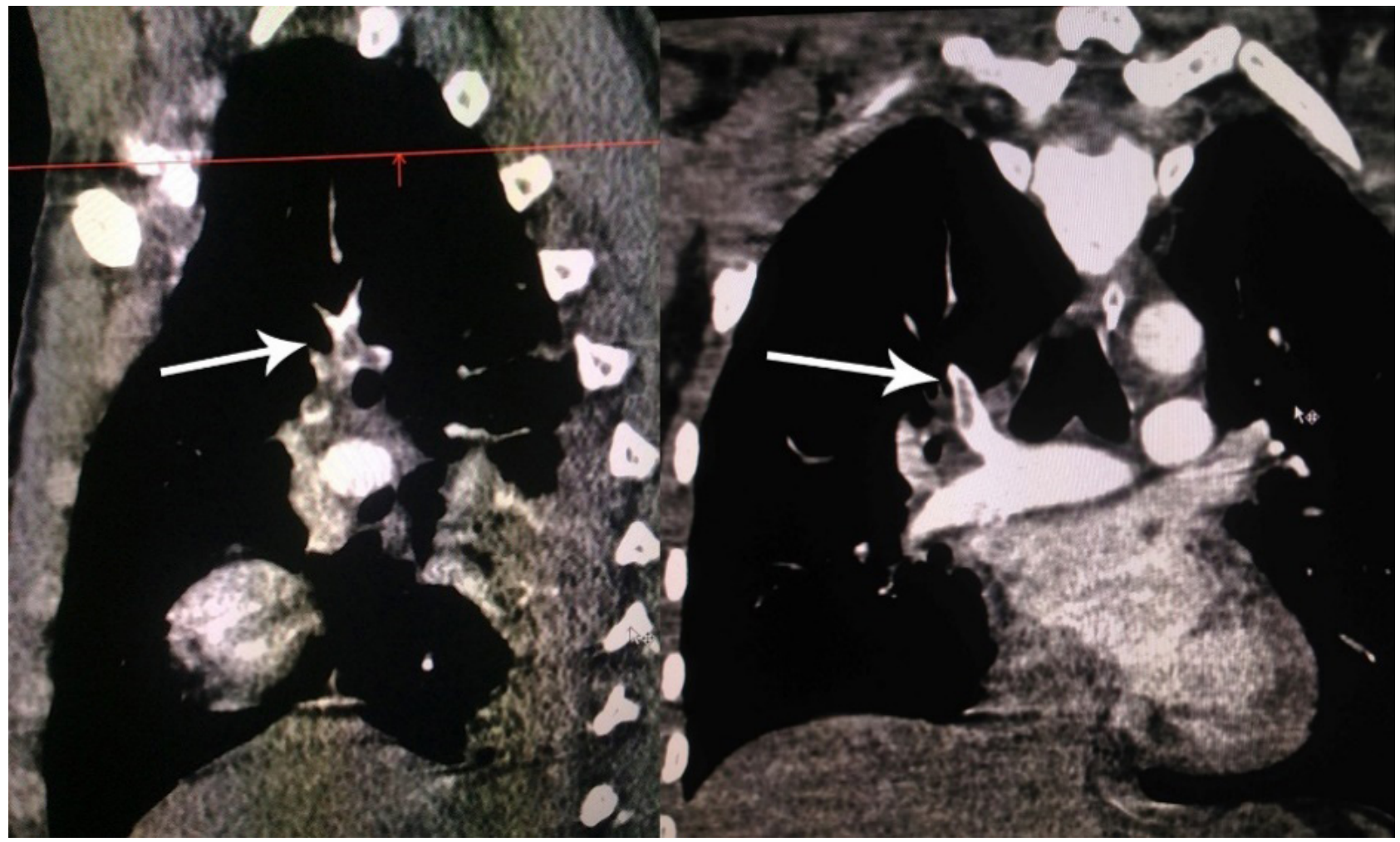

Figure 2: CTPA images showing thrombus in the right upper pulmonary artery (white arrow) 


\section{Discussion}

This is an unusual case in which both pulmonary thromboembolism and fat embolism syndrome occurring in the same patient within 72 hours of a traumatic event. Post-traumatic fat embolism was first reported in 1862 (2) and was defined by Gurd and Wilson in 1970 (3). It is widely accepted that medullary fat from fractured long bones is released into the circulation and ends up obstructing the pulmonary vasculature (4). Our patient fulfilled the three major Gurd and Wilson criteria, which include petechial rash, respiratory insufficiency and cerebral involvement. He also had tachycardia, thrombocytopenia and fever, fulfilling another three minor criteria. His symptoms and signs upon presentation scored 11 in Schonfeld's criteria (5) and fulfilled all four of Lindeque's criteria (6) for fat embolism syndrome. Tests such as detection of fat globules in the urine and fluid from bronchoalveolar lavage and magnetic resonance imaging of the brain to look for cerebral fat embolism (7) are not available at our centre. However, the diagnosis of fat embolism syndrome is largely clinical, and symptoms such as petechiae and hypoxemia after a long bone fracture are pathognomonic of the condition.

Pulmonary thromboembolism is a differential diagnosis of fat embolism syndrome, which also presents with tachypnea and tachycardia. The Wells' test score of the patient was 1.5 , which indicated a low pre-test probability for pulmonary thromboembolism (8). However, the acute blood loss from four long bone fractures could have caused a hypercoagulable state and contributed to the pulmonary thromboembolism, manifested as dyspnea. D-dimer testing is also not available at our centre. A computed tomography pulmonary angiogram was performed, which revealed the presence of thrombus in the pulmonary artery and confirmed the diagnosis of pulmonary thromboembolism. A lower limb Doppler ultrasound scan was not done in the patient due to multiple fractures and traction devices, but would have supported the diagnosis of pulmonary thromboembolism if positive.

Long bone fractures should ideally be fixed as soon as possible; however, there was a 9-hour delay in this case due to late presentation of the patient and resuscitation at the emergency department. Fat embolism syndrome could have been prevented in this patient if the femur fractures were fixed during the first operation. This would have allowed early mobilisation of the patient and together with earlier initiation of anticoagulation therapy, the occurrence of pulmanory thromboembolism may have been prevented. A lower threshold of suspicion is needed so that preventive strategies would routinely be integrated in the management of patients with multiple long bone fractures.

\section{Conclusion}

Both pulmonary thromboembolism and fat embolism syndrome are potentially fatal, and the presence of respiratory distress in a patient with multiple long bone fractures must not be taken lightly. The treatment for fat embolism syndrome is essentially supportive, thus, after resuscitation and stabilisation, early fixation must be performed to prevent the occurrence of this condition. A low Wells' score does not rule out pulmonary thromboembolism, and acute blood loss can cause hypercoagulability, which may precipitate a thrombotic event, necessitating the use of computed tomography pulmonary angiogram to confirm the diagnosis (9). Preventive measures such as early mobilisation and anticoagulation therapy should be started as early as permissible. Although rare, the coexistence of fat embolism syndrome and pulmonary thromboembolism in the same patient must be considered, especially in patients with multiple long bone fractures, to ensure appropriate management of patients and prevent morbidity and mortality.

\section{References}

1. Parvaiz AK, Feroze A, Showkat A.Gurcoo et al. Fat embolism syndrome in long bone trauma following vehicular accidents: Experience from a tertiary care hospital in north India. Lung India. 2013; 30(2): 97102.

2. Talbot M, Schemitsch EH. Fat embolism syndrome: history, definition, epidemiology. Injury. 2006; 37(4): S3-7.

3. Evert A. Eriksson, Joshua Rickey, Stuart M. Leon et al. Fat embolism in pediatric patients: An autopsy evaluation of incidence and etiology. Journal of Critical Care. 2015; 30(1): 221.e1-221.e5.

4. Gurd AR, Wilson RI. The fat embolism syndrome. J Bone Joint Surg Br. 1974; 56(3): 408-16.

5. Schonfeld SA, Ploysongsang Y, et al. Fat embolism prophylaxis with corticosteroids. A prospective study in high-risk patients. Ann Intern Med. 1983; 99(4): 438-43.

6. Lindeque BGP, Schoeman HS, Dommisse GF, et al. Fat embolism and the fat embolism syndrome. A double-blind therapeutic study. J Bone Joint Surg Br. 1987; 69(1): 128-31.

7. Stienen MN, Gautschi OP. Cerebral fat embolism after severe road traffic accident. The American Journal of Emergency Medicine. 2012; 30(5): 831.e1-831.e2.

8. Wells PS, Anderson DR, Rodger $\mathrm{M}$, et al. Derivation of a simple clinical model to categorize patients probability of pulmonary embolism: increasing the models utility with the SimpliRED D-dimer. Thromb Haemost. 2000; 83(3): 416-20.

9. Levin D, Seo JB, Kiely DG, et al. Triage for suspected acute Pulmonary Embolism: Think before opening Pandora's Box. European Journal of Radiology. 2015; 84 (6): 1202-11. 